\title{
Critical Issues in Designing and Implementing Temporal Analytics
}

\author{
Bodong Chen*1, Simon Knight ${ }^{2}$, Alyssa Friend Wise ${ }^{3}$
}

\begin{abstract}
The importance of temporality in learning has been long established, but it is only recently that serious attention has begun to be paid to the precise identification, measurement, and analysis of the temporal features of learning. From 2009 to 2016, a series of temporality workshops explored temporal concepts and data types, analysis methods for exploiting temporal data, techniques for visualizing temporal information, and practical considerations for the use of temporal analyses in particular contexts of learning. Following from these efforts, this two-part Special Section serves to consolidate research working to progress conceptual, technical and practical tools for temporal analyses of learning data. In addition, in this second and final editorial we aim to make four contributions to the ongoing dialouge around temporal learning analytics to help us move towards a clearer mapping of the research space. First, the editorial presents an overview of the five papers in Part 2 of the Special Section on Temporal Analyses, highlighting the dimensions of data types, learning constructs, analysis approaches, and potential impact. Second, it draws on the fluid relationship between 'analyzed time' and 'experienced time' to highlight the need for caution and criticality in the purposes temporal analyses are mobilized to serve. Third it offers a guide for future work in this area by outlining important questions that all temporal analyses should intentionally address. Finally, it proposes next steps learning analytics researchers and practitioners can take collectively to advance work on the use of temporal analyses to support learning.
\end{abstract}

\section{Keywords}

Temporality, time, temporal learning analytics, impact

Published: 04/09/18

${ }^{1}$ Corresponding author. Email: chenbd@umn.edu Address: Department of Curriculum and Instruction, University of Minnesota, 159 Pillsbury Drive SE, Minneapolis, MN 55455, USA, ORCID: 0000-0003-4616-4353

${ }^{2}$ Email: Simon.Knight@uts.edu.au Address: 15 Broadway, Ultimo, NSW, 2007, Faculty of Transdisciplinary Innovation, University of Technology, Sydney, Australia, ORCID: 0000-0002-8709-5780

${ }^{3}$ Email: alyssa.wise@nyu.edu Address: NYU-LEARN, Learning Analytics Research Network, New York University, 82 Washington Square East, 7th Floor, New York, NY 10003, USA

\section{Introduction}

This issue of the Journal of Learning Analytics contains the second part of the Special Section on Temporal Analyses of Learning Data (the first part can be found in JLA 4[3]). Learning, like many kinds of developmental processes, occurs over time. The importance of temporality in learning has been long established (e.g., Sannino, Daniels, \& Gutiérrez, 2009; Slattery, 1995), but it is only recently that serious attention has begun to be paid to the precise identification, measurement, and analysis of the temporal features of learning. As described in the first guest editorial (Knight, Wise, \& Chen, 2017), this Special Section originated from a series of workshops on temporality that took place over a seven-year period at international conferences on Technology-Enhanced Learning, Learning Sciences, and Learning Analytics. The workshop series bridged research communities concerned with educational, technological, and analytical issues, and it is fitting that the workshop series found an eventual home in Learning Analytics - a multidisciplinary research community operating in the middle space where these concerns intersect. From 2009 to 2016, these temporality workshops explored a wide range of considerations including temporality concepts, temporal data types, analysis methods for exploiting temporal data, techniques for visualizing temporal information, and practical considerations for the use of temporal analyses in particular contexts of learning. Over these years we observed a widening awareness of the importance of temporal considerations and a growing interest in applying temporal analytics to learning data in the service of improving educational practice (Chen, Wise, Knight, \& Cheng, 2016).

In the first guest editorial, we began with the premise that temporal information in learning data is frequently underused and, on the whole, temporal concerns are underexplored in educational research (Knight et al., 2017). We then highlighted a series of conceptual, methodological, and operational challenges that explain the lack of attention to temporality thus far. To 
move towards a clearer mapping of the research space, we reviewed the different kinds of data types, temporal features, analysis approaches, and potential impact represented in the five studies included in Part 1 of the Special Section. Building on this foundation, as well as the temporality workshop series and related efforts in specialized research domains such as self-regulated learning (Molenaar \& Järvelä, 2014), this editorial aims to achieve four goals:

1. Present the five papers in Part 2 of the Special Section, highlighting the dimensions of data types, learning constructs, analysis approaches, and potential impact as we did for the first part of the Special Section

2. Draw on the fluid relationship between 'analyzed time' and 'experienced time' to highlight the need for caution and criticality in the purposes temporal analyses are mobilized to serve

3. Guide future work in this area by outlining important questions that all temporal analyses should intentionally address

4. Offer next steps learning analytics researchers and practitioners can take collectively to advance work in this area

\section{Part Two of the Special Section}

\subsection{Data Types}

As in the first part of this special section, the second set of papers includes several studies which present temporal analysis of discourse data. Oshima, Oshima \& Fujita (this issue) examine transcripts of undergraduates engaged in face-to-face collaborating jigsaw groups as part of a teacher certification program. Chiu (this issue) also examines face-to-face collaboration transcripts, but in a ninth-grade setting where students learning algebra through collaborative problem solving.

In addition, this part of the special section includes analysis of several kinds of temporal data that were not present in the first set of papers. First, Riel, Lawless \& Brown (this issue) work with clickstream data from a self-paced online professional development course for teachers offered in Moodle. Second, Liu, Davenport \& Stamper (this issue) and Mahzoon, Maher, Eltayeby, Dou \& Grace (this issue) both examine ongoing measures of student performance. Liu et al. work in the case of a chemistry virtual lab tutor, focusing on first attempt success of items associated with particular 'knowledge components'. This data stream is also aligned with screen capture and audio data as part of a larger multi-modal approach. Mahzoon et al. work with a variety of grades assigned in an undergraduate computer science course (e.g., for quizzes, labs, tests) as well as background demographic features and student reflections.

Three of these five papers focus on temporal data collected intensely over relatively condensed periods of time; both Oshima et al. and Chiu examined episodes of synchronous collaboration while Liu et al. studied use of the chemistry tutor during two 50-minute class periods. The other two papers examine data collected over a more extended time frame: three weeks for the professional development course in Riel et al. and a full semester for the undergraduate course in Mahzoon et al.

\subsection{Learning Constructs and Analysis Approaches}

The learning constructs and analysis approaches represented in the second part of the Special Section are diverse. Liu et al. are concerned with knowledge components, a learning construct defined as "the skills, concepts, and/or facts required to complete a given task or problem correctly" (p. 43). In the intelligent tutor they study, learning tasks are tagged with related knowledge components, making it possible to examine a learner's performance trajectory with respect to a specific knowledge component. They theorize that the performance trajectory of a knowledge component should ideally only increase over time as learners are given successive opportunities to apply it in tasks. This led them to search for violations of this expectation (i.e., noticeable drops in performance) visually, via negative slope estimates, and through high residual values. These are then considered 'focal' moments requiring further fine-grained analysis of the aligned audio and screen capture data.

Mahzoon et al. are interested in predicting learning success in a degree program, a construct of larger magnitude and time frame. To aid this prediction task, Mahzoon et al. present a novel approach to using temporal features in multi-year heterogeneous student data. Their work conceptualizes a student's learning journey as a sequence model of course performance data. They generate coefficients from the sequence model to represent the student's temporal learning journey, and then include these generated temporal coefficients as features in predictive models of learning success. This study presents a useful example of developing temporal features that can be incorporated into existing data mining approaches.

Riel et al. focus on the distribution of learning efforts in self-paced online courses, which touch upon issues related to learner control and self-regulation behaviours. Instead of treating temporal features as being secondary to another analysis, they develop two new statistics to index aspects of temporality. In particular, they propose measures to characterize the timing of how a learner completes their coursework in terms of a (1) timing index (defined as the point at which a participant has devoted $50 \%$ of the total effort or time they will put into a course), and (2) spacing count (the percent of regular time intervals in the overall course timeframe during which work was performed). Using these two derived measures, Riel et al. venture further to predict teacher self-efficacy after completing the course. Such attempts to derive new temporal measures of practical and theoretical values are needed. 
Both Oshima et al. and Chiu are concerned with constructs related to learning dialogue in groups. Chiu is interested in the relationship between domain knowledge and social metacognition involved in collaborative problem solving, specifically whether the correct evaluation of mathematical ideas followed by correct new mathematical ideas is a commonly occurring sequence and one that is linked to higher-level learning outcomes. He compares the examination of these questions in the forward direction at the outcome level (i.e., does a 'Higher overall proportion of the evaluation $\rightarrow$ New idea' sequence predict the group's solution score?) and in the backward direction at the process level (i.e., in groups with higher solution scores, are new correct ideas more likely to follow correct evaluations?). In contrast, Oshima et al. are concerned with shared epistemic agency in jigsaw group activities. To measure this, they used a network analysis method to characterize how the semantic space of discourse is socially shared among learners. By mapping out a network centrality measure over time, they could then identify pivotal contributions in group discourse for further qualitative analysis. A similar network analysis method was applied to identifying promising ideas in a paper from the first part of the Special Section (Lee \& Tan, 2017).

\subsection{Potential Impact}

Across the papers insights into learning are offered, with their potential for supporting learning flagged. As Mahzoon et al., highlight, predictive models may be enriched by temporal dependency data. Such data can then be used for data storytelling that describe learning journeys to support sensemaking. In their analysis, then, learning must be seen - and may be impacted at - multiple outcome levels (from individual course to full program), and data storytelling is a means through which to achieve impact. Understanding learner trajectories is also key in two other papers. In Riel et al., we see the complexity of understanding self-paced learning, and how analysis of log data can be translated into visualizations to show 'early starters', 'late starters', and consistent workers. These visualizations could be used by course designers to understand patterns of participation both for redesign and personalization purposes. In contrast, Liu et al.'s work is situated within an intelligent tutoring system. Their temporal analysis is grounded in their understanding of an idealised learning trajectory, against which drops in performance can be assessed. Temporal analysis, then, is used ato target further fine-grained analysis (using multi-modal data), to gain deeper insights into the learning process, in this case by researchers or learning designers. As the authors note, the potential of such tools is also to provide a way for teachers to extract real examples of students based on temporal features of their learning trajectories. A similar approach is taken by Oshima et al., who identify pivotal contributions in order to target further analysis of productive interaction. By using this mixed-methods approach, they suggest that the time taken to analyze group interaction may be reduced, and thus the results of such analysis can be used to support educators in their learning design. Finally, and also with a focus on student dialogue, Chiu's work provides an approach for the analysis of discourse sequences and their relation to outcome, offering potential new insights to researchers. Additionally, this analysis holds potential to educators to intervene in student learning processes, through assessing those process and their relationship to outcome.

\section{Analyzed Time Shapes Experienced Time}

Increasing interest in and calls for temporal analyses of learning have arisen in part due to the growing availability of finegrained temporal data generated by increasingly sophisticated technologies that allow for moment-by-moment tracking of our every click, utterance, gesture, and gaze. Looking back in history, such advances in the precision with which time is measured have continually been associated with shifts in how societies conceive of time. The relationship between 'perceived time' (that which is experienced by humans as part of a lived event) and 'mechanical time' (that which is measured and can thus be dissociated from human events) was described almost a hundred years ago by Mumford (1934) who noted that the invention of the clock as a device for measurement also produced the ideas of 'seconds' and 'minutes' which in turn became actors that structured human activity. Thus, while temporal analyses primarily draw on mechanical time (and fine-grained measurement, analysis and control of it), we need to problematize impacts of temporal analyses and analytics on experiences of time in learning.

Education has been shaped repeatedly by the increasingly precise measurement of time. Since the industrial age, the age of a learner has been the primary determinant of where they are placed in school. This implies strong embedded assumptions about the similarity of developmental trajectories and rates as which people learn, and through the development of grade-level curricula, the sequence in which they should do so. Time also creates a framework for dividing learning experiences into smaller and smaller pieces: the calendar year into quarters, a quarter into weeks, and a day into teaching blocks. Within a single class session, careful sequencing of content and detailed planning down to the minute are often championed. The rise of digital learning environments takes things even further as we can easily record timestamped learner activities down to the second or even millisecond. However, we must always question whether our ability (and that of the learning technologies we build) to measure time in increasingly precise ways is actually being leveraged to examine learning in commensurately more sophisticated ones.

In addition, while increased precision of measured time offers the possibility of finer planning, monitoring, and control of educational relationships, such decisions need to be made thoughtfully with regard to learning outcomes. In particular, reflexivity in how, why, and for what purposes we constrain temporal expectations is urgently needed, as is attention to potential negative consequences of over-structuring activity to predetermined schedules. Thus, we emphasize that researchers 
need to consider theoretical entailments of temporal analyses and the ways in which they may impact learning activity as integral to their analytics development process.

\section{Guiding Questions for Temporal Considerations in Learning Analytics}

Learning analytics as a field offers unprecedented opportunities for critically deepening our thinking and practice related to temporality in education. In the previous guest editorial, we reviewed learning constructs, data types, and analysis approach es, in light of conceptual, methodological, and pragmatic challenges that were flagged as important dimensions in the development of temporal analytics (Knight et al., 2017). In the current editorial we build on this foundation by posing a series of questions intended to foster robust temporal learning analytics. The questions highlight important theoretical, empirical, and practical dimensions that situate "temporal analyses in the larger frame of learning analytics efforts to understand and improve learning.

Q1: What are the key learning constructs and how are they conceptualized with respect to time?

Q2: Where are the learning constructs observed and how are they represented in data?

Q3: How are the theorized learning constructs analyzed with regard to their temporal features?

Q4: To whom and how do the learning analytics provide temporal insight that can lead to temporal impact on learning processes?

These questions can be used in three complementary ways to guide the:

1. Development of learning analytics - by aligning learning constructs with temporal features and operationalizing these in ways that provide insight into and impact on learning

2. Evaluation of learning analytics - with respect to the operationalization of temporal features, learning constructs, and the relationship between them

3. Presentation of learning analytics — by offering a framework through which to explicate important assumptions about time and temporality that would otherwise remain implicit

\section{Q1: What are the key learning constructs and how are they conceptualized with respect to time?}

Learning analytics is clearly focused on 'learning' (Gašević, Dawson, \& Siemens, 2015), yet we do not always agree on what learning is and which specific constructs of learning merit attention. A move to consider temporal learning analytics foregrounds the need to not only articulate the learning constructs of interest but also how they are theorized to manifest temporally. In the education literature, constructs refer to conceptual entities that are theorized to be important for learning, but are not directly observable. Some learning constructs are individual-cognitive, such as one's domain-specific knowledge and skills. The construct of knowledge components in Liu et al. is an example. Other learning constructs at the individual level are social-emotional, for example one's emotional expressiveness, emotional knowledge, or emotional regulation (Denham, 2006). Of course, these learning constructs do not exist independently from each other. For instance, cognition and emotion can be deeply intertwined, leading researchers to investigate learners' cognitive-affective states such as boredom, frustration, and delight (Baker, D’Mello, Rodrigo, \& Graesser, 2010). Self-regulated learning involves a combination of learning constructs that are cognitive, metacognitive, and motivational (Sperling, Howard, Staley, \& DuBois, 2004). In group settings, learning constructs expand beyond the individual to include group-level constructs such as socially shared regulation and collaborative intersubjectivity (Malmberg, Järvelä, \& Järvenoja, 2017). In temporal analytics, we first need to identify and articulate the learning constructs of interest in a study in order to have a theoretical basis from which to conceptualize the relevant temporal features.

Moving from learning constructs to temporal features, two common kinds of temporal features are often considered in theorizing learning constructs with respect to time. The first relates to the passage of time, considering questions such as when a construct is seen to manifest, how frequently or for how long it occurs, or by which rate the construct changes over time. For example, at a basic level we can examine how much time a learner spends on learning tasks and when a learning behaviour occurs (Riel et al.,). In other settings we are interested in how frequently something happens; for example, the intensity with which learners use video controls (e.g., play, pause, seek) while watching a MOOC video (Kim et al., 2014). Other work has considered the pace of change, for example examining the growth rate of performance for a knowledge component (Liu et al.,) or the decay rate of cognitive-affective states over a session of conversational dialogue with an intelligent tutor (D'Mello \& Graesser, 2011).

A second common kind of temporal feature is concerned with temporal order, considering questions about how events or states are sequentially organized (Reimann, 2009). Importantly, analyses focused on temporal order generally ignore differences in the duration between events, and thus can present a very different picture of the learning activity (Wise \& Padmanabhan, 2009). A consideration of temporal order can include attention directly to which events commonly follow others (either directly or after some lag), rates of transition from one state to another, similarities in sequences, or the presence of higher level phases of activity. For instance, the construct of self-regulated learning is theorized as a cyclical process of planning, monitoring, and evaluation/adaptation. Temporal features relating to the sequence in which these events occur is thus 
a central concern in providing a rich conceptual account of self-regulated learning (Winne, 2014). Wise and Chiu (2011) consider sequence-oriented features in the context of knowledge construction at two levels. At the meso-level they examine implied temporality in terms of whether online discussions actually occurred in coherent segments of activity progressing from lower to higher knowledge construction phases (as theory would suggest). At the micro-level they probed into the kinds of posts immediately preceding any changes in the dominant phase of discussion and the relationship of the knowledge construction phase of an individual post to the kinds of contributions made in the two preceding posts, thereby probing relationships between individuals in a collaborative setting.

It is important to note that these two types of temporal features are often considered together. In the study of regulation in collaborative learning, for example, researchers are interested in both how each regulation process (e.g., monitoring) changes over time and how one regulation process may sequentially facilitate another (Malmberg et al., 2017). Moreover, temporal features can be considered at varying time scales (e.g., semester, week, session), and at different levels (e.g., individual, group), which can lead to the creation of novel composite features.

To summarize, carefully conceptualizing a particular learning construct in relation to time and available temporal features lays a theoretical foundation for analysis. It is only through the investigation of the relevant theorized temporal features of learning constructs that we can unleash the full potential of temporal data and analyses. In doing so we make explicit key analytical decisions - choices such as whether to focus on durations, changes over time, or event sequences - as well as the integration between temporality and the targeted cognitive, affective, and social constructs. We suggest that by articulating these relationships, colleagues developing and evaluating learning analytics can more readily interrogate alignment between methods and theory across diverse contexts. Such work is important in developing accounts of temporality in learning. Without it, temporal analyses are simply another addition to the toolbox of learning analytics techniques that may offer additional new 'curiosities,' but don't contribute to developing a systematic understanding of learning (Wise \& Shaffer, 2015). The suggested approach takes a proactive and grounded stance to analysis, rather than starting from thinking about what temporal information is available in data. This is important in the development of theoretically aligned learning analytics, and in considering (and critiquing) why particular approaches to operationalization have been taken and any compromises required.

\section{Q2: Where are the learning constructs observed and how are they represented in data?}

There are a number of concerns regarding data in developing learning analytics based on temporal features of learning. In order to analyze a construct, we must first identify contexts in which it may be meaningfully observed. These contexts may introduce constraints onto our research and data. We must also develop a clear operationalization of the kind of data needed to analyze the particular construct and its temporal components, and how we will analyze that data while respecting temporal features. Some of this context is fundamentally related to the learning construct under analysis; for example, whether the learning goal is group or individual learning, will fundamentally be bound up with learning contexts in which that learning occurs. However, other features of context can have important impact on the learning, while providing varied lenses onto a construct; for example, in group work, although some features of synchronicity may be fundamentally constrained by the construct, others - such as the scheduling frequency and duration of meetings - are not. Moreover, in different pedagogic and assessment settings, target learning constructs may manifest rather differently (Knight, Buckingham Shum, \& Littleton, 2014). For example, the way that self-regulation manifests in MOOCs, in which videos and quizzes can be watched at will, may be rather different to that in a typical lecture-based course. Yet, these contexts have important implications for how we understand the learning. As Winne (2014) notes "characteristics of data shape choices about analyzing data, a researcher needs to probe how features of questions about SRL [or other learning constructs] can be manifested in data" (p. 229).

Of course, often in learning analytics work we are constrained not only by practical context, but through the use of preexisting datasets rather than original data collection in contexts over which we have control. In these cases, the question is 'What analytics can be used that respect the temporal features of the learning construct?', rather than 'What data can be gathered from the learning context, given the construct to be probed, and how will it be analyzed?'. Importantly, even when analysis is based on an existing dataset, consideration should be given to the nature of that data and how it operationalizes its target constructs. As Wise and Shaffer (2015) note, there is a temptation to use sophisticated methods even where the data is not suitable (for a range of reasons), or the approach is not theoretically justified. This can be particularly true in cases where we work with existing datasets over which we have little control. However, theory provides us the tools to address the question 'Where is the learning construct observed, and how is it represented in data?' in a reasoned way, and can support us in subsequent interpretation of analysis for impact (Wise \& Shaffer, 2015). In addition, theory can help us to understand how to relate specific contexts (individual classrooms, learning tasks, etc.) to our constructs, and consider the kinds of data we might obtain about that learning.

Importantly, this is also true of treatment of temporal features. For example, we might observe data as sequences of events, or as events that occur with separating intervals. On occasion, times intervals may be precisely equal and can be treated as equivalent. However, at other times we may choose to treat events separated by different intervals as equivalent despite their different temporal spacing, and at other times, we may wish to treat such data differently. At times, these constraints are flexible, while in other cases, such as in analysis of tasks that must be completed within a single school period/class session, 
they may not be. Education is replete with such temporal constraints, such as the duration of class periods, progression through grades or university levels, and project trajectories through learning levels (such as common core standards in the US, or national curriculum levels in the UK).

Much of the data on which learning analytics researchers draw is derived from computer systems. Indeed, technological changes have been a key factor in raising interest in, and potential for, temporal analyses of learning. While the range of tools and data structures is rapidly expanding, nonetheless particular systems hold implications for the granularity of temporal data obtained, the modalities across which it is obtained, and 'shapes' in which it is stored (e.g. LMS logs, discourse structures, multimodal 'streams', etc.). At times, temporal data is readily available in these systems in a usable form. Other times, work is required to aggregate or normalize data, for example as in Riel et al., normalizing varied timelines to create a comparable timescale, or aggregating activities that occur within a particular window (minutes of each other, a certain number of turns of talk, etc.) into single events.

Moreover, some temporal constructs are derived from other data; for example, using time-stamped activities to derive periodicity, and relate that to self-regulation of learning. Data selection and transformation that is grounded in theorization of learning constructs and considerations of learning contexts, can drive our selection and application of temporal analyses.

\section{Q3: How should the theorized learning constructs be analyzed with regard to their temporal features?}

This two part Special Section has demonstrated a variety of temporal analysis techniques applied in various settings. While this editorial does not permit a comprehensive review of temporal analyses, below we anchor our discussion of Q3 in one example to show how the choice of analysis method derives from the earlier discussed issues.

The example we present is situated in an elementary school following a unique pedagogy known as knowledge building (Chen, Resendes, Chai, \& Hong, 2017). A central goal of knowledge building is to help students collectively take cognitive responsibility for continual improvement of their ideas. In other words, the students need to decide for themselves which lines of inquiry to pursue, which ideas are worth spending time on, what gaps need to be filled in their knowledge-building discourse, etc. This leads to interest in two particular learning constructs: productivity of student discourse indicated by idea improvement, and the sequences of epistemic moves made by students to achieve this. The data available was online, asynchronous discourse recorded in a knowledge-building environment known as Knowledge Forum. Face-to-face discourse took place in classrooms but was not captured in this particular study. Student discourse was coded for epistemic moves (24 in 6 major categories) which characterize different ways elementary students contribute to collective knowledge building. With the goal of distinguishing productive discourse (that which included idea improvement) based on sequences of epistemic moves, Chen et al. (2017) applied two widely adopted temporal analysis approaches-lag-sequential analysis and frequent sequence mining. In doing so, they were able to identify common sequential patterns associated with productive dialogues. In particular these two approaches offered complimentary insights into discourse patterns related to constructive use of information and sustained efforts to problematize existing explanations. It is illuminating in this case that sequential patterns of epistemic moves were predictive of group productivity, while earlier attempts at prediction based on summary statistics (aggregated numbers of different epistemic moves) fell short.

While the temporal analysis approaches adopted in this study were focused on the order of events (in this case epistemic moves in discourse), understanding could be further enriched by integrating other temporal analysis methods. For instance, the socio-semantic-temporal network analysis introduced in Oshima et al. has already been used to characterize 'rotating leadership' among individual students in knowledge-building discourse (Ma, Matsuzawa, \& Scardamalia, 2016). While both studies examine how a network centrality measure change over time, Ma et al. focuses on tracking 'leadership moments' of individual students, whereas Oshima et al. examine changes of 'shared epistemic agency' at the group level. These two exemplifications of an examination of change over time both provide potential further analyses for the knowledge-building discourse in Chen et al., (2017). These two investigations of change over time exemplify potential additional temporal analyses for the knowledge-building discourse in Chen et al. (2017).

While encouraging the exploration of temporal analysis methods, we also caution that applying a temporal analysis method proven useful elsewhere to a different context requires substantial conceptual work. For example, to adopt the sequence model approach in Mahzoon et al. in the study of epistemic moves in discourse, important theoretical issues and analytical choices need to be addressed. Key questions may include 'whether there is an idealized sequence model of discourse moves in knowledge-building discourse', and if so, 'on what theoretical or pedagogical basis, 'at which level (individual, small group, or community)', and 'in what timeframe'? While we should seek novel analysis methods or novel applications of established methods, the analysis needs to be grounded in careful consideration of learning constructs and temporal features, and careful documentation, justification, and interrogation of temporal analytical decisions.

\section{Q4: To whom and how do the learning analytics provide temporal insight that can lead to temporal impact on learning processes?}

Finally, temporal learning analytics foreground an important issue that should be of concern for all analytics: understanding impact. We deploy learning analytics because we wish to produce change in learning, and as discussed above change always occurs over time. To achieve this goal, as a field learning analytics needs to focus on moving beyond just providing insight 
into learning to also offer ways to impact on that learning, recognising too and that this process is itself temporal in nature. Of course, initial phases of developing learning analytics for impact may well involve developing and deriving insight from tools whose end-users are researchers, and we recognize the importance of this foundational work. However, to produce changes in learning, achieving impact in authentic learning contexts must be in our sights, and learning analytics researchers should be concerned with the actionability of their work from the start of the process.

Thus, we distinguish two important questions for evaluating learning analytics (temporal or otherwise):

1. What insight does the analytic provide into learning? In this question we include concerns regarding evaluation of the analytics (including the preceding questions), and their validity.

2. What impact can the analytic have on learning? In this question, we include concerns regarding the pedagogic potential of the analytics, how they have been deployed thus far, the theory of change, and the 'integrity' of the analytical system (how well does it communicate with regard to risks, uncertainties, or ambiguities) (Buckingham Shum, 2016)?

In addition, we distinguish a weak and strong sense of temporal 'impact'. In the weak sense, impact is always temporal simply in the sense that for learning analytics to achieve impact, it must achieve change, and this is not typically the result of one-shot overnight interventions, but occurs over time (Wise, Zhao \& Hausknecht, 2014). In a strong sense, impact itself can be modelled as change over time. This offers the opportunity for theorization of second-order temporality that describes how the process by which temporal learning constructs themselves evolve. For example, if we want to go from condensed activity to distributed, we must address the question of what the intermediate stages between those patterns look like.

In summary, to achieve impact learning analytics researchers must generate insight not only about the nature of learning constructs, but also with regard to implementation, integration, and communication of learning analytics within contexts. That is, learning analytics is about learning, but to have impact on learning we need to understand more than just learning constructs in the abstract. Learning analytics must develop accounts of how learning analytics can be designed around learning constructs, built into learning contexts, and communicated or activated within those contexts to achieve some change for a targeted population. Learning analytics must then develop approaches to communicate for impact. In the case of temporal analytics this includes attention to: (1) how temporal descriptions are communicated, for example, through visualizations of state changes over time; (2) how temporal prescriptions are communicated, for example, 'you did A, now you should do B'; and (3) how the changes produced by such communication can themselves be tracked over time. The conceptualization and documentation of these important decisions are critical for developing impact through temporal learning analytics.

\section{Conclusions: Ways Forward in Time}

Learning occurs over time. This two-part Special Section has highlighted research that explicitly examines temporal features in the analysis of learning. Building on these wide ranging contributions, in this editorial we have flagged the significance of the emerging body of work on temporal learning analytics, and the potential for their analyses to shape our experience of learning over time. In developing, evaluating, and presenting learning analytics, we have offered four guiding questions:

Q1: What are the key learning constructs and how are they conceptualized with respect to time?

Q2: $\quad$ Where are the learning constructs observed and how are they represented in data?

Q3: How are the theorized learning constructs analyzed with regard to their temporal features?

Q4: To whom and how do the learning analytics provide temporal insight that can lead to temporal impact on learning processes?

To develop as a field, learning analytics must theorize temporality in learning constructs and articulate their place in the collection and analysis of data, and more broadly, socio-technical and socio-political systems where learning takes place. While tools and techniques for the analysis of temporal data are increasingly available, temporal learning analytics must ground itself in the well theorized application of analytic techniques to learning data.

Beyond the scope of temporal learning analytics, many of the ideas brought into sight through the work in this space demonstrate needs in the field of learning analytics more broadly. The research in this two-part Special Section have highlighted some important concerns in the analysis of learning relating to context, theory, data, analysis, and their alignment. Finally, echoing Gašević, Dawson, and Siemens (2015), we conclude this editorial with an important reminder: Learning analytics are about learning. But let's not forget, learning analytics aren't just about learning, they're about impacting learning.

\section{Declaration of Conflicting Interest}

The author(s) declared no potential conflicts of interest with respect to the research, authorship, and/or publication of this article. 


\section{Funding}

The author(s) declared no financial support for the research, authorship, and/or publication of this article.

\section{References}

Baker, R. S. J. d., D’Mello, S. K., Rodrigo, M. M. T., \& Graesser, A. C. (2010). Better to be frustrated than bored: The incidence, persistence, and impact of learners' cognitive-affective states during interactions with three different computer-based learning environments. International Journal of Human-Computer Studies, 68(4), 223-241. https://doi.org/10.1016/j.ijhcs.2009.12.003

Buckingham Shum, S. (2016). Algorithmic accountability for learning analytics. Presented at the Institute of Education \& London Knowledge Lab, University College London. Retrieved from http://simon.buckinghamshum.net/2016/03/algorithmic-accountability-for-learning-analytics/

Chen, B., Resendes, M., Chai, C. S., \& Hong, H.-Y. (2017). Two tales of time: uncovering the significance of sequential patterns among contribution types in knowledge-building discourse. Interactive Learning Environments, 25(2), 162175. https://doi.org/10.1080/10494820.2016.1276081

Chen, B., Wise, A. F., Knight, S., \& Cheng, B. H. (2016). Putting temporal analytics into practice. In Proceedings of the Sixth International Conference on Learning Analytics \& Knowledge - LAK'16 (pp. 488-489). New York, New York, USA: ACM Press. https://doi.org/10.1145/2883851.2883865

Denham, S. A. (2006). Social-Emotional Competence as Support for School Readiness: What Is It and How Do We Assess It? Early Education and Development, 17(1), 57-89. https://doi.org/10.1207/s15566935eed1701_4

D’Mello, S., \& Graesser, A. (2011). The half-life of cognitive-affective states during complex learning. Cognition \& Emotion, 25(7), 1299-1308. https://doi.org/10.1080/02699931.2011.613668

Epp, C. D., Phirangee, K., \& Hewitt, J. (2017). Talk with Me: Student Pronoun Use as an Indicator of Discourse Health. Journal of Learning Analytics, 4(3), 47-75. https://doi.org/10.18608/jla.2017.43.4

Gašević, D., Dawson, S., \& Siemens, G. (2015). Let's not forget: Learning analytics are about learning. TechTrends, 59(1), 64-71. https://doi.org/10.1007/s11528-014-0822-x

Kim, J., Guo, P. J., Cai, C. J., Li, S.-W. (daniel), Gajos, K. Z., \& Miller, R. C. (2014). Data-driven Interaction Techniques for Improving Navigation of Educational Videos. In Proceedings of the 27th Annual ACM Symposium on User Interface Software and Technology (pp. 563-572). New York, NY, USA: ACM. https://doi.org/10.1145/2642918.2647389

Knight, S., Buckingham Shum, S., \& Littleton, K. (2014). Epistemology, assessment, pedagogy: where learning meets analytics in the middle space. Journal of Learning Analytics, 1(2), 23-47. http://doi.org/10.18608/jla.2014.12.3

Knight, S., Wise, A. F., \& Chen, B. (2017). Time for change: Why learning analytics needs temporal analysis. Journal of Learning Analytics, 4(3), 7-17. https://doi.org/10.18608/jla.2017.43.2

Lee, A. V. Y., \& Tan, S. C. (2017). Promising Ideas for Collective Advancement of Communal Knowledge Using Temporal Analytics and Cluster Analysis. Journal of Learning Analytics, 4(3), 76-101. https://doi.org/10.18608/jla.2017.43.5

Ma, L., Matsuzawa, Y., \& Scardamalia, M. (2016). Rotating leadership and collective responsibility in a grade 4 Knowledge Building classroom. International Journal of Organisational Design and Engineering, 4(1-2), 54-84. https://doi.org/10.1504/IJODE.2016.080159

Malmberg, J., Järvelä, S., \& Järvenoja, H. (2017/4). Capturing temporal and sequential patterns of self-, co-, and socially shared regulation in the context of collaborative learning. Contemporary Educational Psychology, 49, 160-174. https://doi.org/10.1016/j.cedpsych.2017.01.009

Molenaar, I., \& Järvelä, S. (2014). Sequential and temporal characteristics of self and socially regulated learning. Metacognition and Learning, 9(2), 75-85. https://doi.org/10.1007/s11409-014-9114-2

Mumford, L. (1934). Technics and civilization (pp. 145-215). London, UK: Routledge \& Kegan Paul Ltd.

Reimann, P. (2009). Time is precious: Variable- and event-centred approaches to process analysis in CSCL research. International Journal of Computer-Supported Collaborative Learning, 4(3), 239-257. https://doi.org/10.1007/s11412009-9070-Z

Sannino, A., Daniels, H., \& Gutiérrez, K. D. (2009). Activity theory between historical engagement and future-making practice. In A. Sannino, H. Daniels, \& K. D. Gutiérrez (Eds.), Learning and expanding with activity theory (pp. 1-15). Cambridge: Cambridge University Press.

Slattery, P. (1995). A Postmodern Vision of Time and Learning: A Response to the National Education Commission Report Prisoners of Time. Harvard Educational Review, 65(4), 612-634. https://doi.org/10.17763/haer.65.4.0908t56382151541

Sperling, R. A., Howard, B. C., Staley, R., \& DuBois, N. (2004). Metacognition and Self-Regulated Learning Constructs. Educational Research and Evaluation: An International Journal on Theory and Practice, 10(2), 117-139. https://doi.org/10.1076/edre.10.2.117.27905 
Winne, P. H. (2014). Issues in researching self-regulated learning as patterns of events. Metacognition and Learning, 9(2), 229-237. https://doi.org/10.1007/s11409-014-9113-3

Wise, A. F., \& Chiu, M. M. (2011). Analyzing temporal patterns of knowledge construction in a role-based online discussion. International Journal of Computer-Supported Collaborative Learning, 6(3), 445-470. https://doi.org/10.1007/s11412-011-9120-1

Wise, A., \& Padmanabhan, P. (2009). Developing structural-temporal visualizations of asynchronous threaded online learning conversations. In Proceedings of E-Learn World Conference on E-Learning in Corporate, Government, Healthcare, and Higher Education (pp. 3253-3261). Vancouver, BC: Association for the Advancement of Computing in Education (AACE).

Wise, A. F., \& Shaffer, D. W. (2015). Why theory matters more than ever in the age of big data. Journal of Learning Analytics, 2(2), 5-13. https://doi.org/10.18608/jla.2015.22.2

Wise, A., Zhao, Y., \& Hausknecht, S. (2014). Learning analytics for online discussions: Embedded and extracted approaches. Journal of Learning Analytics, 1(2), 48-71. http://doi.org/10.18608/jla.2014.12.4 